\title{
Spin State of Chloroquine-Heme Complexes: Formation of a Hemin Tetramer Adduct
}

\author{
Kai F. Schwedhelm ${ }^{1}$, Martin Horstmann ${ }^{1}$, Johan H. Faber ${ }^{2}$, Yana Reichert ${ }^{2}$, Michael Büchner ${ }^{2}$, \\ Gerhard Bringmann ${ }^{*}, 2$ and Cornelius Faber ${ }^{*}, 1$ \\ ${ }^{I}$ Department of Experimental Physics 5, Am Hubland, University of Würzburg, Germany \\ ${ }^{2}$ Institute of Organic Chemistry, Am Hubland, University of Würzburg, Germany
}

\begin{abstract}
Complex formation between the antimalarial drug chloroquine and its presumed target ferriprotoporphyrin IX in three different solutions $(\mathrm{pH} 6.5, \mathrm{pH} \mathrm{9}$, and in a water methanol mixture) is characterized by nuclear magnetic resonance, UV spectroscopy, and mass spectrometry. NMR paramagnetic relaxation measurements are used to derive intermolecular distances between the molecules and model structures of the complexes are calculated by molecular dynamics simulations. Observation of an unusual spin state in NMR measurements leads to the postulation of a novel 4:2 stoichiometry of the complex, which is supported by mass spectrometry and UV spectroscopy.
\end{abstract}

\section{INTRODUCTION}

Malaria as an infectious disease is responsible for more than 500 million incidences and a death toll of 1.3 million [1] every year, mainly in children under five years of age in tropical regions worldwide. The most commonly used antimalarial drug is chloroquine (CQ) [2, 3], but adverse reactions and growing resistance especially of the most dangerous parasite Plasmodium falciparum urgently demand for the development of novel antimalarial agents.

The presumed target of many antimalarial drugs is ferriprotoporphyrin IX (FPIX, heme), a moiety of human hemoglobin, which is released during the blood stage of a malaria infection inside the human red blood cells. These drugs were proposed to prevent hemozoin crystal formation from FPIX through direct binding to free heme, which then exerts strong oxidative stress on the food vacuole membranes of Plasmodium and eventually kills the malaria parasite.

An essential step in the development of new agents is a detailed investigation of the molecular target, FPIX, and the associated mode of action of well known antimalarial drugs.

Ferriprotoporphyrin IX is known to be present in multiple molecular states in solution, for example hematin $\left(\mathrm{H}_{2} \mathrm{O} / \mathrm{OH}-\mathrm{Fe}(\mathrm{III}) \mathrm{PPIX}\right), \overline{\mathrm{A}}$-oxo-dimer (two FPIX monomers bridged by an Fe-O-Fe bond), chelat dimer or crystalline . hematin [4-6].

The first atomic resolution structures of FPIX in complex with several antimalarial drugs as determined by NMR were presented by De Dios and co-workers [7]. The technique was further refined in our group [8] to additionally include the determination of effective correlation times of the complexes.

\footnotetext{
*Address correspondence to these authors at the (CF) Department of Experimental Physics 5, Am Hubland, University of Würzburg, Germany; E-mail: faber@physik.uni-wuerzburg.de and (GB) Institute of Organic Chemistry, Am Hubland, University of Würzburg, Germany;

E-mail: bringmann@chemie.uni-wuerzburg.de
}

In this article, the formation of a novel chloroquine-FPIX tetramer adduct is proposed from measurements of an unusual spin state in FPIX using nuclear magnetic resonance spectroscopy. The results are supported by mass spectrometry and UV spectroscopy. In addition, atomic resolution structures of the proposed complexes in three different solvents are obtained from NMR paramagnetic relaxation measurements.

\section{MATERIALS AND METHODS}

Classic NMR experiments for structure determination are futile if the system of interest contains paramagnetic ions (like Fe(III) in FPIX), which cause severe line broadening and signal degradation. However, the paramagnetic effect itself is dependent on the distance between the paramagnetic center and the proton spins in the complex and can be used as source of structural information.

It is described by the Solomon-Bloembergen equation [9]

$$
r=\left(\frac{2}{15}\left(\frac{\mu_{0}}{4 \pi}\right)^{2} \frac{\hbar^{2} \gamma_{I}^{2} \gamma_{S}^{2} S(S+1)}{R_{\text {complex }}}\left(\frac{\tau_{C}}{1+\left(\omega_{S}-\omega_{I}\right)^{2} \tau_{C}^{2}}+\frac{3 \tau_{C}}{1+\omega_{I}^{2} \tau_{C}^{2}}+\frac{6 \tau_{C}}{1+\left(\omega_{S}+\omega_{I}\right)^{2} \tau_{C}^{2}}\right)\right)^{\frac{1}{6}}
$$

which contains three essential parameters: the longitudinal relaxation rate $R_{\text {complex }}$ of each proton resonance in the NMR spectrum, the effective correlation time $\tau_{C}$ of the complex, and the spin state $S$ of the paramagnetic ion. If all parameters are known, the distance between the Fe center in FPIX and individual protons in the drug molecule can be calculated with high precision. $R_{\text {complex }}$ can be measured using standard NMR inversion recovery pulse sequences. The effective correlation time $\tau_{C}$ can be derived from repeated experiments at different $B_{0}$ fields, subsequently fitting Equation 1 to the results. The spin state $S$ is accessible using the "Evans method". A detailed description of the entire process can be found in $[7,8]$.

Ferriprotoporphyrin IX chloride (FPIX), chloroquine diphosphate salt (CQ), 2,2,6,6-tetramethylpiperidine 1-oxyl (TEMPO), 3-(trimethylsilyl)propionic acid- $\mathrm{d}_{4}$ sodium salt 
(TSP), phosphate buffered saline (PBS), $5 \mathrm{~mm}$ NMR tubes, and WILMAD coaxial inserts were purchased from SigmaAldrich Chemie $\mathrm{GmbH}$ (Munich, Germany). Deuterium oxide $\left(\mathrm{D}_{2} \mathrm{O}\right)$, methan- $\mathrm{d}_{1}$-ol $(\mathrm{MeOD})$, and deuterium sodium oxide (NaOD) were obtained from Euroisotop (Saarbrücken, Germany).

For determination of the complex stoichiometry, aliquots were mixed in predetermined [FPIX]/[CQ] ratios, while keeping the total concentration of the two species constant at 2 $\mathrm{mM}$ and $56 \overline{\mathrm{A} M}$ for NMR and UV experiments, respectively.

\section{UV Spectroscopy}

UV spectroscopy was performed on a Varian Cary 50 spectrophotometer (Varian, Inc., Palo Alto, CA, USA). The absorbance was measured at $365 \mathrm{~nm}$ in standard $1 \mathrm{~cm}$ cuvettes ( $1.5 \mathrm{ml}$ sample volume).

For binding studies aliquots of a stock solution of CQ $(0.168 \mathrm{mM})$ were added to a stock solution of FPIX-Ā-oxo dimer (8.4 $\overline{\mathrm{AM}}$ ) in a buffered solution ( $\mathrm{pH} 6.5$ and $\mathrm{pH} 9)$. The complexation was monitored by the decrease of the UV absorbance at $\lambda=365 \mathrm{~nm}$. Final spectra were recorded about $10 \mathrm{~min}$ after each addition, when sequentially recorded spectra varied by less than 0.001 absorbance units. Digested titration data were corrected for dilution and the corresponding binding isotherm was then analyzed using a nonlinear curve fitting with a 1:1 association model (CQ:FPIX-Ā-oxo dimer).

The stoichiometry of the FPIX:CQ complex was determined through NMR and UV spectroscopy using the Job plot representation as described in the literature [10,11].

\section{NMR Spectroscopy - Spin State Measurement}

The FPIX molecule contains a central, paramagnetic $\mathrm{Fe}(\mathrm{III})$ ion. The spin state $\mathrm{S}$ of this ion can be determined using a method proposed by Evans [12]. The chemical shift of any given $\mathrm{H}$ atom resonance in the spectrum depends on the bulk susceptibility $\chi_{0}$ of the surrounding medium. From $\chi_{0}$ the magnetic moment $\bar{A}$ and subsequently the spin state $S$ of FPIX can be calculated [13, 14]. Magnetic coupling between the two $\mathrm{Fe}$ ions is considered weak and can therefore be neglected [15].

A coaxial NMR tube system (WILMAD, WilmadLabGlass, Buena, NJ, USA) was used to determine the difference in chemical shift [16] of a reference solution (inner tube) compared to the sample solution (outer tube).

The inner capillary (reference solution) contained $100 \mu \mathrm{l}$ of a $1 \mathrm{mM}$ TSP solution in $\mathrm{D}_{2} \mathrm{O}$ with PBS $(10 \mathrm{mM}$, $\mathrm{pH} 6.5$ or 9) in all experiments that were performed in aqueous solution. For determination of the geometry factor the outer capillary contained $1 \mathrm{mM}$ TSP and the standard radical molecule TEMPO at a concentration of $2 \mathrm{mM}$. For the spin state determination experiments aliquots of the aqueous stock solutions of FPIX and of CQ were mixed in Eppendorf tubes to give a concentration in the final sample of $1.0 \mathrm{mM}$ FPIX (dimer) and predetermined titration steps of CQ in the range of zero to eight molar equivalents. A stock solution of TSP in $\mathrm{D}_{2} \mathrm{O}(100 \mathrm{mM})$ was prepared, and aliquots thereof were added to the samples to give a final concentration of 1 mM TSP. A solution of $\mathrm{D}_{2} \mathrm{O}$ with PBS (10 mM, pH 6.5 or 9) was added to give a total sample volume of $500 \mu$ l. Argon was gently bubbled through the samples for $30 \mathrm{~min}$. to eliminate the paramagnetic oxygen impurities. Subsequently $300 \mu \mathrm{l}$ of the samples were transferred to the outer tube of a coaxial tube system. Samples in $\mathrm{MeOD} / \mathrm{D}_{2} \mathrm{O}$ were prepared accordingly.

Samples were placed in a $5 \mathrm{~mm}$ NMR tube, reference solutions were filled in a capillary, which was placed in the central axis of the NMR tube. 1D ${ }^{1} \mathrm{H}$ NMR spectra of each sample were acquired on a Bruker DMX 600 (14.1 T) spectrometer and the frequency shift difference of the HOD solvent peaks and of TSP in the two compartments was measured $(\Delta f)$, yielding the same results. The geometry factor $k$ was calibrated through measurements of the standard radical molecule ( $n=1)$ 2,2,6,6-tetramethylpiperidine-1-oxyl (TEMPO).

\section{NMR Spectroscopy - Determination of Relaxation Rates and Effective Correlation Time}

Phosphate buffered saline (PBS) (pH 7.4) was dissolved in $\mathrm{D}_{2} \mathrm{O}(0.1 \mathrm{M})$ and adjusted to the desired $\mathrm{pH}$ by $\mathrm{HCl}(0.5$ M) or $\mathrm{NaOH}(0.5 \mathrm{M})$. When adjusted the solution was evaporated to dryness and subsequently dissolved in $\mathrm{D}_{2} \mathrm{O}$ to give a final concentration of $10 \mathrm{mM}$. Stock solutions (40 $\mathrm{mM}$ ) of CQ were prepared in $\mathrm{D}_{2} \mathrm{O}$. Stock solutions of FPIX $(15.0 \mathrm{mM}$, dimer) were prepared in $50 \mathrm{mM} \mathrm{NaOD}$ and used within $60 \mathrm{~min}$. Samples were prepared in Eppendorf tubes.

For relaxation rate measurements aliquots of the CQ and FPIX stock solutions were mixed in predetermined ratios to give a final concentration of $2.0 \mathrm{mM} \mathrm{CQ}$ and $0-0.2 \mathrm{mM}$ FPIX dimer. A solution of $\mathrm{D}_{2} \mathrm{O}$ with PBS $(10 \mathrm{mM}, \mathrm{pH} 6.5$ or $\mathrm{pH}$ 9.0) was added to give a final sample volume of $800 \mu \mathrm{l}$, which was transferred into $5 \mathrm{~mm}$ NMR tubes. For the experiments in $\mathrm{MeOD} / \mathrm{D}_{2} \mathrm{O}$ aliquots of a FPIX stock solution (15 mM, dimer) in MeOD and of the aqueous CQ stock solution $(40 \mathrm{mM})$ were mixed in the same predetermined ratios. The samples were subsequently diluted with a $1: 3$ mixture of $\mathrm{MeOD}$ and $\mathrm{D}_{2} \mathrm{O}$ with PBS (10 mM, pH 6.5) to give a final sample volume of $800 \mu \mathrm{l}$ and then transferred into $5 \mathrm{~mm}$ NMR tubes.

${ }^{1} \mathrm{H}$ NMR spectra were recorded at six different spectrometers - three different magnetic field strengths for each sample. FPIX:CQ (pH 6.5) was measured on a Bruker Avance 400 (9.4 T), a Bruker DMX 600 (14.1 T), and a Bruker Avance 750WB (17.6 T) spectrometer. FPIX:CQ (pH 9) and FPIX:CQ $\left(\mathrm{MeOD} / \mathrm{D}_{2} \mathrm{O}\right)$ were measured on a Bruker Avance 400 (9.4 T), a Bruker Avance 600 (14.1 T), and a Bruker Avance 800 (18.8 T) spectrometer (Bruker Biospin $\mathrm{GmbH}$, Rheinstetten, Germany). Sample temperature was 299 K. Drug peaks were assigned in accordance to the literature [17] and confirmed using COSY experiments. No changes in drug chemical shifts were observed in any of the samples. FPIX resonances were strongly broadened and could not be assigned.

Strong signal broadening caused by high concentrations of paramagnetic FPIX in the samples was overcome using a technique proposed by Leed et al. [7]. NMR relaxation rates for each resonance in every sample of a dilution series (FPIX:CQ ratios 0:1, 1:100, 1:80, 1:50, 1:20, 1:10) were measured. The values were extrapolated to a $1: 1$ ratio and from the slope of a linear fit, the relaxation rate $R_{\text {complex }}$ was calculated. 
The effective correlation time $\tau_{C}$ for each resonance was calculated by fitting Eq. (1) to the relaxation rates $R_{\text {complex }}$ obtained from measurements at three different magnetic field strengths. Fitting procedures were performed using MATHEMATICA Version 5 (Wolfram Research, Champaign, IL, USA).

\section{Mass Spectrometry}

For MS experiments, stock solutions of FPIX (2.5 mM, dimer) in $\mathrm{MeOH}: \mathrm{H}_{2} \mathrm{O}(3: 1 \mathrm{vol} / \mathrm{vol})$ and of $\mathrm{CQ}(5 \mathrm{mM})$ in $\mathrm{MeOH}: \mathrm{H}_{2} \mathrm{O}$ (3:1 vol/vol) were prepared immediately prior to the measurements. CQ and FPIX were mixed in a molar concentration ratio 1:1 starting from the stock solutions, and subsequently diluted with $\mathrm{H}_{2} \mathrm{O}$ and $\mathrm{MeOH}$ to give a concentration in the final sample of $250 \mu \mathrm{M}$ CQ and $125 \mu \mathrm{M}$ FPIX-Ā-oxo-dimer in $\mathrm{MeOH}: \mathrm{H}_{2} \mathrm{O}$ (3:1 vol/vol). The solutions had final $\mathrm{pH}$ values of ca. 7. Identical samples were prepared using MeOD: $\mathrm{D}_{2} \mathrm{O}$.

Electrospray ionization time-of-flight (ESI-TOF) mass spectra were measured with a Bruker Daltonics micrOTOF focus (Bruker Daltonics, Bremen, Germany) mass spectrometer equipped with an ESI ion source (Apollo). A stainlesssteel spraying capillary and, as a transfer capillary, a nickelcoated glass capillary with an inner diameter of $500 \overline{\mathrm{A}}$ were utilized. The ions were generated continuously by introducing the sample solutions into the ion source with a syringe pump (Cole Palmer Instruments 789100C) at a flow rate of $4 \overline{\mathrm{A}} \mathrm{l} / \mathrm{min}$. Mild conditions were applied by using the following parameters: Capillary voltage $-4.6 \mathrm{kV}$; end plate voltage: $-4.1 \mathrm{kV}$; exit voltage: $110 \mathrm{~V}$; skimmer voltage: 60 $70 \mathrm{~V}$; hexapol RF: $400 \mathrm{~V}$; Set Flight tube: $9000 \mathrm{~V}$; Set Corrector Fill: 56 V; Set Pulsar Pull 406 V; Set Pulsar Push: $406 \mathrm{~V}$; Transfer Time: 89 Ās; Pre Puls Storage: 20 Ās; temperature of drying gas: $50^{\circ} \mathrm{C}$. The dry gas flow was 2.0 $1 / \mathrm{min}$ and the nebulizer pressure was 0.5 bar. Some parameters had a significant influence on signal intensities. The vacuum in the TOF analyzer was $3.12 \cdot 10^{-7}$ mbar. The mass range was $m / z 200-3000$. The calibration standard was introduced after the sample measurement. Calibration was performed as an internal calibration with a 1:100 dilution of ES Tuning Mix (G2421A; Agilent) in 95\% acetontrile / 5\% water with $m / z 622,922,1522,2122$ by using the quadratic regression mode.

For the measurement 24 scans were averaged to improve the signal-to-noise ratio. Due to the isotopic distribution over a broad $\mathrm{m} / \mathrm{z}$ region caused by $\mathrm{Fe}$ and $\mathrm{Cl}$, the intensity of the signal for the lowest-mass isotopic composition $(\mathrm{X}+0)$ was too small in intensity for an accurate mass measurement. Therefore, typically the most intense signal $(X+n)$ of this isotopic distribution was taken and compared with the respective calculated value. For calculation of the corresponding mass values of the isotopic distribution, the software modul "Bruker Daltonics IsotopePattern" of the software Compass 1.1 from Bruker Daltonik $\mathrm{GmbH}$, Bremen, Germany was used.

\section{Molecular Modeling}

Intermolecular distances between $\mathrm{Fe}(\mathrm{III})$ in FPIX and individual $\mathrm{H}$ atoms in $\mathrm{CQ}$ were obtained by evaluating the Solomon-Bloembergen equation, Eq. (1), for every signal in the CQ NMR spectrum. These distances were used as constraints for molecular dynamics simulations of the FPIX:CQ complex using the XPLOR-NIH version 2.17.0 software package [18]. 30 least-energy models were selected and overlaid and used for further analysis using the PyMOL software [19].

\section{RESULTS}

\section{UV Spectroscopy}

Complex formation between FPIX and CQ was characterized by UV spectroscopy. Job plot experiments showed that a complex was formed with a molar ratio of two FPIX molecules per one CQ. The dissociation constant $K_{D}$ was determined as $(3.9 \pm 0.2) \cdot 10^{-6} \mathrm{M}$ and $(4.1 \pm 0.4) \cdot 10^{-6} \mathrm{M}$ at $\mathrm{pH} 6.5$ and $\mathrm{pH} 9$, respectively. These values agree well with previously published values of $3.16 \cdot 10^{-6} \mathrm{M}$ and $(2.5 \pm$ $1.8) \cdot 10^{-6} \mathrm{M}[20-22]$.

\section{NMR Spin State Measurement}

The spin state of $\mathrm{Fe}$ in FPIX was determined from measurement of the bulk susceptibility. Fig. (1) shows the calculated number $n$ of free electrons per Fe as a function of the relative amount of $\mathrm{CQ}$ in aqueous solution at $\mathrm{pH} 6.5$ and $\mathrm{pH} 9$ and in $\mathrm{MeOD} / \mathrm{D}_{2} \mathrm{O}$. In all three solutions $n$ was between 2.6 and 3.1 for free FPIX, which indicated a spin state of $S=3 / 2$. In both buffered solutions a significant change in the $\mathrm{Fe}$ spin state was observed upon addition of CQ. For an excess of CQ, $n$ quickly leveled off at values of $n$ $=1.4$ and $n=1.0$ at $\mathrm{pH} 6.5$ and $\mathrm{pH} 9$, respectively. Both values clearly evidenced a spin state of $S=1 / 2$. In $\mathrm{MeOD} / \mathrm{D}_{2} \mathrm{O}$, by contrast, no change in the number of free electrons was observed. Regardless of the amount of drug added, $n$ remained nearly constant at approximately $n=2.6$, which suggested a spin state of $S=3 / 2$.

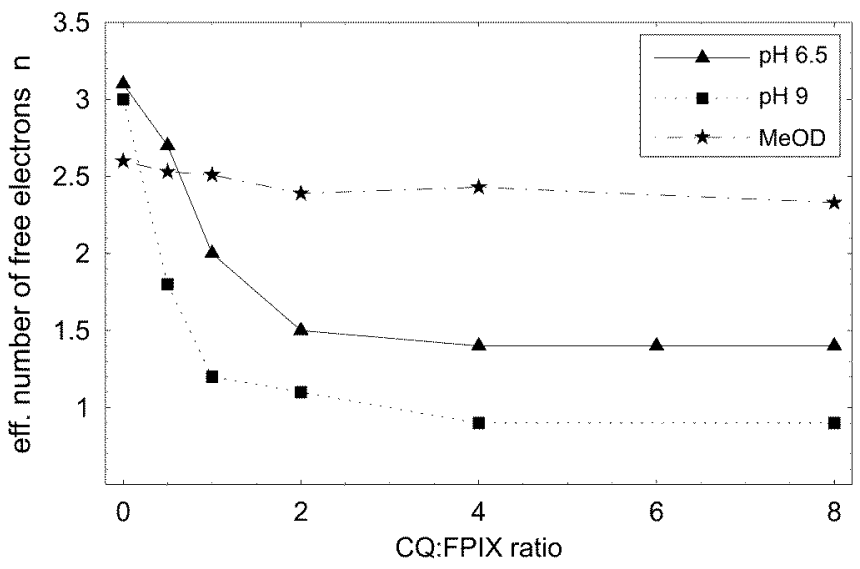

Fig. (1). Determination of the spin state. Plot of the effective number of free electrons $n$ versus the concentration of CQ, for FPIX:CQ at pH 6.5 ( ), FPIX:CQ at $\mathrm{pH} 9$ (. ), and FPIX:CQ in $\mathrm{MeOD} / \mathrm{D}_{2} \mathrm{O}(*)$.

The Job plot representation of the measured frequency shift $\Delta f$ is shown in Fig. (2). The maximum at a CQ:FPIX ratio of 0.63 (dashed line) indicated a nominal stoichiometry of one CQ molecule per one FPIX-Ā-oxo dimer (2:1 with respect to FPIX monomer). This result perfectly corroborated the above findings from UV spectroscopy.

To explain the observed stoichiometry and the changing spin state upon addition of CQ, we propose a novel model: 


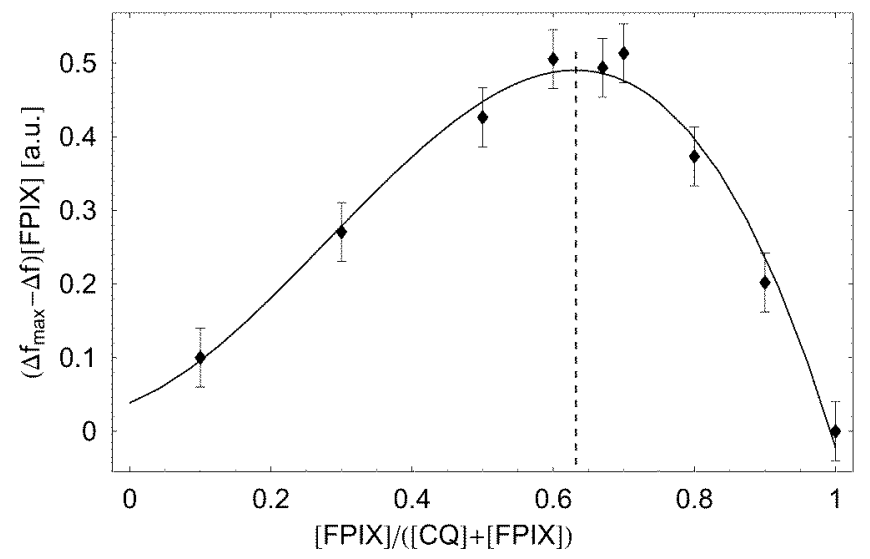

Fig. (2). Determination of the complex stoichiometry. Job plot of the frequency splitting $\Delta f$ between sample and reference solution versus CQ:FPIX ratio in the samples. The maximum of the curve is at a ratio of 0.63 (dashed line) indicating a stoichiometry of one CQ per two FPIX monomers in the FPIX:CQ complex.

FPIX in solution is present as a tetramer (i. e., as a dimer of two A-oxo dimers) which binds two CQ molecules. The Fe of one monomer subunit of each $\bar{A}$-oxo dimer are mutually coordinated by one carboxy group of the corresponding monomer subunit of the second $\bar{A}$-oxo dimer (Fig. 3). An illustration of the 4:2 complex is shown in the supporting information (Fig. S3). Such a complex is explained by the ligand field theory [23]. All Fe atoms are octahedrally complexed. The inner atoms $\mathrm{Fe}^{(\mathrm{b})}$ and $\mathrm{Fe}^{(\mathrm{c})}$, bridging the two $\bar{A}$-oxo dimers, are each coordinated by the four nitrogens of the FPIX tetrapyrrole, the bridging oxygen of the $\bar{A}$-oxo dimer, and a carboxy group from the other FPIX-Ā-oxo dimer. This leads to a strong splitting $\Delta O$ of the $t 2 g$ and $e g$ molecular orbitals, leading to a low-spin configuration of the Fe with a spin state $S=1 / 2$. For the Fe atoms of the outer monomer subunits, the sixth coordination site is occupied by a water molecule. The weaker ligand forces only a small splitting $\Delta O$, which leads to a high spin configuration of $\mathrm{S}=$ $5 / 2$. The average spin state of the tetramer observed by NMR spectroscopy therefore is $S=3 / 2$. Upon binding of $C Q$, the stronger ligand forces a larger splitting of molecular orbitals. The increased splitting changes the spin state from $S=5 / 2$ (high spin) to $S=1 / 2$ (low spin), resulting in an average spin state of $S=1 / 2$ for the entire tetramer FPIX:CQ complex. The absolute stoichiometry of this system is FPIX:CQ $4: 2$, which is consistent with our results from NMR and UV experiments.

\section{Mass Spectrometry}

Independent evidence for a 4:2 complex of FPIX:CQ was provided by MS analysis. In the mass spectrum of FPIX:CQ in a methanol water mixture, both, species with four and two porphyrin systems, were detected (Fig. 4). In the spectra, the isotopic distribution of the doubly protonated [CQ-FPIX-OFPIX $_{2}-\mathrm{O}$-FPIX-CQ+2H] $]^{2+}$ was overlapped by the isotopic distribution of the single protonated [FPIX-O-FPIX-CQ+1H] ${ }^{+}$. The difference between the experimental $\mathrm{m} / \mathrm{z}$ (1569.03806) and the calculated $\mathrm{m} / \mathrm{z}$ value $(1569.04111)$ for the $[\mathrm{X}+9]$ of the doubly protonated CQ-FPIX-O-FPIX 2 -O-FPIX-CQ was 1.9 ppm. Additionally, the isotopic distribution of [CQ-FPIX-OFPIX $_{2}$-O-FPIX-CQ+2H] ${ }^{2+}$ agreed well with the calculated isotopic distribution of $\left[\mathrm{C}_{172} \mathrm{H}_{182} \mathrm{Cl}_{2} \mathrm{Fe}_{4} \mathrm{~N}_{22} \mathrm{O}_{18}\right]^{2+}$. This confirmed the existence of the tetramer FPIX:CQ complex CQ-FPIX-O-FPIX ${ }_{2}$-O-FPIX- CQ in solution.
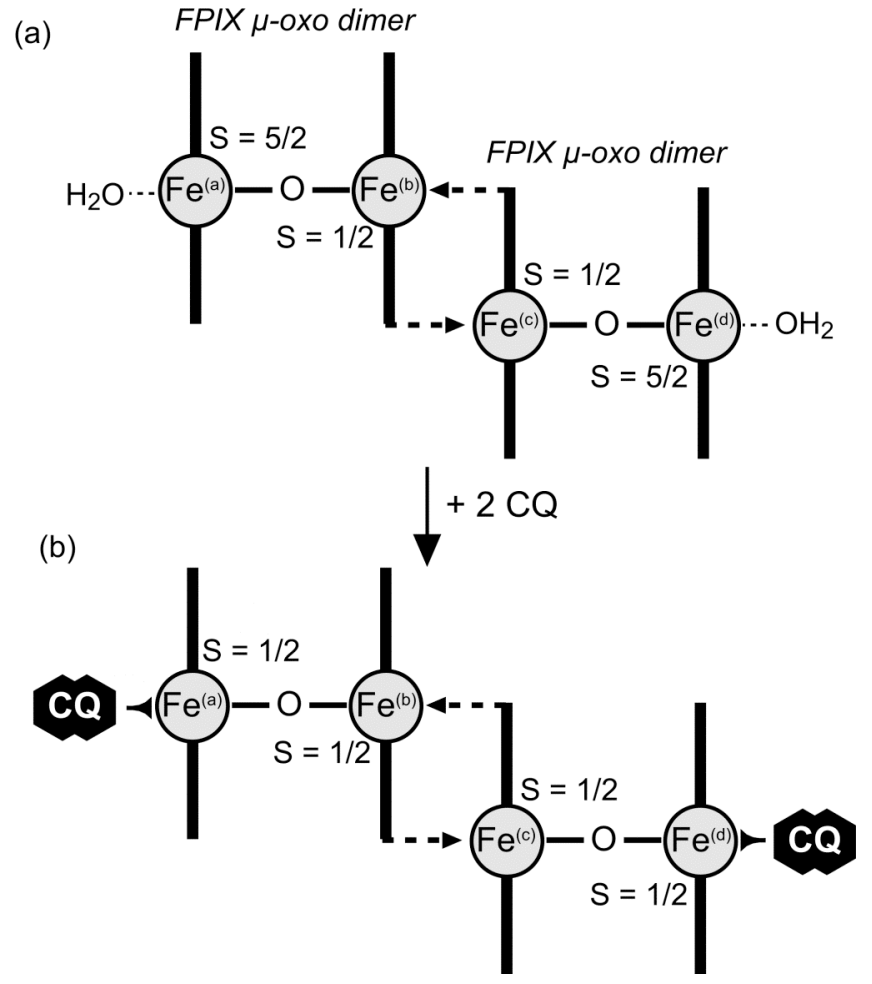

Fig. (3). Schematic model of the FPIX:CQ complex. Two FPIX Aoxo dimers are bridged via carboxy groups (dashed arrows) mutually coordinating $\mathrm{Fe}^{(\mathrm{b})}$ and $\mathrm{Fe}^{(\mathrm{c})}$. Spin states of the $\mathrm{Fe}$ atoms are indicated. (a) FPIX tetramer in aqueous solution with complexed $\mathrm{H}_{2} \mathrm{O}$ bound to the outer atoms $\mathrm{Fe}^{(\mathrm{a})}$ and $\mathrm{Fe}^{(\mathrm{d})}$. The average spin state observed with NMR is $S=3 / 2$. (b) Upon addition of CQ to the samples, $\mathrm{CQ}$ replaces $\mathrm{H}_{2} \mathrm{O}$ at the outer atoms $\mathrm{Fe}^{(\mathrm{a})}$ and $\mathrm{Fe}^{(\mathrm{d})}$, thus leading to a change in $\mathrm{Fe}$ spin state. The average spin state observed with NMR is $S=1 / 2$.

In a separate experiment, a solution of $250 \bar{A} \mathrm{M}$ in $\mathrm{MeOD} / \mathrm{D}_{2} \mathrm{O}$ (vol/vol 3:1) was used. In the tetrameric complex, there were four carboxy groups in each FPIX $\bar{A}$ oxo dimer, which were subject to H/D exchange and one exchange site in each drug molecule (CQ N-H). In conjunction with the double protonation/deuteration during the MS experiments, this yielded a total of twelve H/D exchange sites. The observed degree of H/D exchange $(6 \mathrm{~m} / \mathrm{z}$ in the double charged complex) corresponded perfectly to the expected value.

Furthermore, the isotope distribution at ca. $\mathrm{m} / \mathrm{z} 1575$ $\left(\left[\mathrm{C}_{172} \mathrm{H}_{170} \mathrm{Cl}_{2} \mathrm{Fe}_{4} \mathrm{~N}_{22} \mathrm{O}_{18} \mathrm{D}_{12}\right]^{2+}\right)$ for the $\mathrm{X}+9$ again confirmed the existence of the complex CQ-FPIX-O-FPIX 2 -O-FPIXCQ (see Supporting Information Fig. (S1)). Other complexes found in the MS analysis were: $[\mathrm{FPIX}]^{+}(\mathrm{m} / \mathrm{z} 614)$, $[2 \mathrm{CQ}+\mathrm{H}]^{+}(m / z 639),[\mathrm{CQ}-\mathrm{FPIX}]^{+}(\mathrm{m} / z$ 993 $),\left[\mathrm{FPIX}_{2}+\mathrm{H}\right]^{+}$ $(m / z \text { 1231), [FPIX-O-FPIX+H] }]^{+}\left(m / z \text { 1249), [CQ-FPIX }{ }_{2}+\mathrm{H}\right]^{+}$ $(\mathrm{m} / \mathrm{z}$ 1550).

\section{NMR Relaxation Measurements}

The 1D ${ }^{1} \mathrm{H}$ NMR spectrum of CQ showed ten resolved signals. These were assigned as shown in Fig. (5). For each of the ten signals, the relaxation rate $R_{\text {complex }}$ and the effective correlation time $\tau_{C}$ were derived (see supporting information 
Tables S1-S3). Largest values for $R_{\text {complex }}$ were obtained at $\mathrm{pH}$ 9. At $\mathrm{pH} 6.5$ and in $\mathrm{MeOD} / \mathrm{D}_{2} \mathrm{O}$ relaxation rates were smaller. However, due to the exponent of $1 / 6$ in Eq. (1) this only translated into a small variation of the intermolecular distances. The calculated values for $\tau_{C}$ were $(1.11 \pm 0.46) \bullet$ $10^{-10} \mathrm{~s}$ at $\mathrm{pH} 6.5,(1.09 \pm 0.60) \cdot 10^{-10} \mathrm{~s}$ at $\mathrm{pH} 9$, and $(1.5 \pm$ $1.1) \cdot 10^{-11} \mathrm{~s}$ for $\mathrm{MeOD} / \mathrm{D}_{2} \mathrm{O}$.
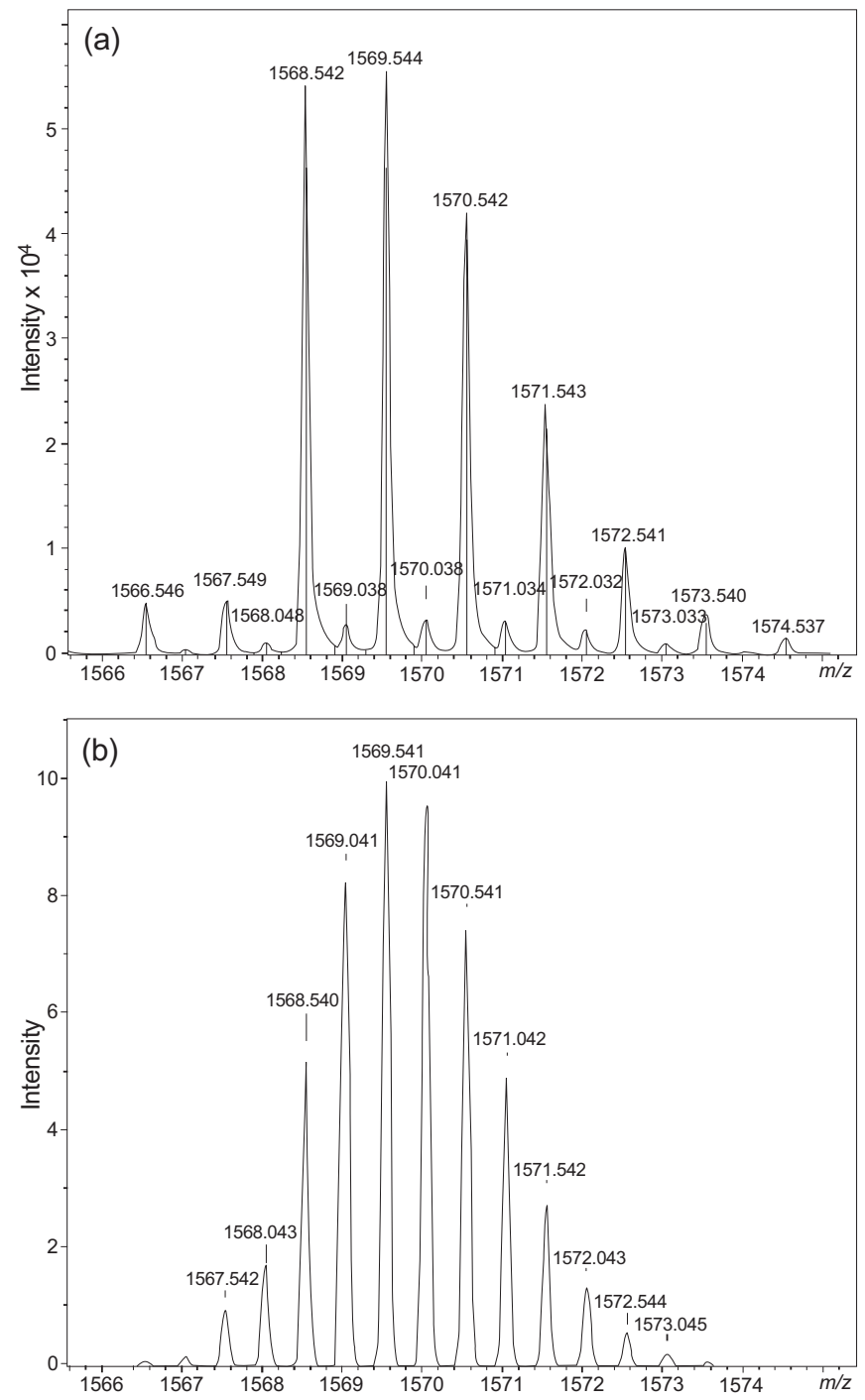

Fig (4). (a) ESI mass spectrum of a $250 \overline{\mathrm{AM}}$ methanol/water (3:1 $\mathrm{vol} / \mathrm{vol}$ ) solution of the CQ:FPIX mixture. The isotope distribution at $\mathrm{m} / \mathrm{z} 1569.038$ reveals the formation of the doubly protonated tetramer complex CQ-FPIX-O-FPIX ${ }_{2}$-O-FPIX-CQ. Signals originating from the tetramer have lower intensity. Every other peak is overlapped by the more intense signals of the twofold protonated dimer. (b) Calculated isotopic distribution of [CQ-FPIX-O-FPIX 2 -O-FPIX$\mathrm{CQ}+2 \mathrm{H}]^{2+}$, corresponding to the elemental composition $\left[\mathrm{C}_{172} \mathrm{H}_{182} \mathrm{Cl}_{2}\right.$ $\left.\mathrm{Fe}_{4} \mathrm{~N}_{22} \mathrm{O}_{18}\right]^{2+}$.

From the three parameters $\left(\tau_{C}, S, R_{\text {complex }}\right)$ for each resonance, the intermolecular distances between the metal center in FPIX and the corresponding hydrogen atoms in CQ were calculated using Eq. (1). Resonances no. 1 to 5 corresponded to the $\mathrm{H}$ atoms in the quinoline moiety of $\mathrm{CQ}$, resonances no. 6 to 10 result from atoms in the CQ side chain. All derived distances are summarized in Table 1. At
pH 9, distances were between 4.5 and $7.2 \AA$, at $\mathrm{pH} 6.5$ between 5.6 and $8.9 \AA$, and in $\mathrm{MeOD} / \mathrm{D}_{2} \mathrm{O}$ between 5.6 and $9.5 \AA$.

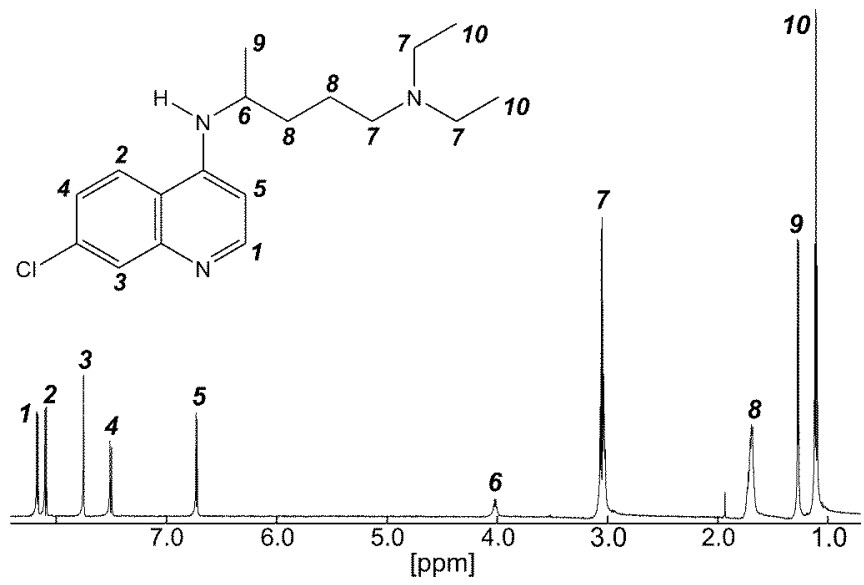

Fig. (5). ${ }^{1} \mathrm{H}$ NMR spectrum of CQ (2mM, pH 6.5) acquired at 750 $\mathrm{MHz}$. Signal assignment is indicated in bold numbers.

The distances were used as restraints in molecular dynamics (MD) calculations, which produced a set of $3 \mathrm{D}$ models for the FPIX:CQ complexes. Since the 4:2 complex was assumed to be perfectly symmetric and each $\bar{A}$-oxo dimer acts as an independent CQ binding site, only one drug molecule in complex with one $\bar{A}$-oxo dimer was simulated to reduce complexity of the calculations. Fig. (6) shows overlays of the seven lowest-energy structures for each complex.

In both buffered solutions (Fig. 6a,b), the quinoline moiety of CQ was aligned nearly parallel to the FPIX tetrapyrrole. The distances and angles between the two planes were $3.6 \AA$ and 25 degrees, and $3.2 \AA$ and 5 degrees at $\mathrm{pH} 6.5$ and $\mathrm{pH} 9$, respectively. CQ was not located centrally above the Fe atom, but slightly shifted towards the rim of the FPIX tetrapyrrole. No covalent or hydrogen bonding interactions were observed. The FPIX Ā-oxo dimer rotated freely in multiples of $90^{\circ}$ around the $\mathrm{Fe}-\mathrm{O}-\mathrm{Fe}$ axis, making interactions between CQ and the side chains of FPIX unlikely. The most obvious difference between the two complexes is the orientation of the CQ side chain. While it extended away from the center and likewise wraped around the tetrapyrrole at $\mathrm{pH} 6.5$, it was aligned along the rim of the tetrapyrrole at $\mathrm{pH}$ 9. In $\mathrm{MeOD} / \mathrm{D}_{2} \mathrm{O}$ the complex structure was less well defined (Fig. 6c), indicating a weaker interaction. The quinoline moiety of CQ was at a distance of $4.3 \AA$ and was tilted by an angle of approximately 40 degrees with respect to the tetrapyrrole plane.

\section{DISCUSSION}

We have used UV, NMR, and MS methods to characterize complex formation between FPIX and CQ. Based on our results, we have proposed a 4:2 stoichiometry of the FPIX:CQ complex, in which two CQ molecules bind to either side of a double FPIX $\bar{A}$-oxo dimer. The driving forces of complex formation are $\pi-\pi$ molecular recognition interactions, hydrophobic interactions, and ligand-field splitting. Since the FPIX A-oxo dimer is a precursor in FPIX detoxification, the 4:2 complex may give a possible hint at the antimalarial mode of action of CQ. 
Table 1. Fe-H Distances Calculated with Eq. (1) and Distances Derived from Final Structures Calculated by Molecular Dynamics Simulations. a) Peaks 1 and 2 for FPIX:CQ (pH 9) were Not Resolved in the NMR Spectrum and Were Discarded in the Following Calculations

\begin{tabular}{|c|c|c|c|c|c|c|}
\hline \multirow[t]{2}{*}{ Atom } & \multicolumn{2}{|c|}{$\begin{array}{c}\mathbf{r}[\AA ̊] \pm 0.7 \AA \\
\text { FPIX:CQ }(\mathbf{p H ~ 6 . 5 )}\end{array}$} & \multicolumn{2}{|c|}{$\begin{array}{c}\mathbf{r}[\AA]] \pm 0.7 \AA \\
\text { FPIX:CQ }(\mathbf{p H} 9)\end{array}$} & \multicolumn{2}{|c|}{$\begin{array}{c}r[\AA] \pm 0.7 \AA \\
\text { FPIX:CQ }\left(\mathrm{MeOD} / \mathrm{D}_{2} \mathrm{O}\right)\end{array}$} \\
\hline & Measured & MD Sim. & Measured & MD Sim. & Measured & MD Sim. \\
\hline 1 & 5.79 & 5.69 & $(4.81)^{\mathrm{a}}$ & $(5.72)^{\mathrm{a}}$ & 6.50 & 6.07 \\
\hline 2 & 5.89 & 5.25 & $(4.81)^{\mathrm{a}}$ & $(3.50)^{\mathrm{a}}$ & 5.89 & 6.46 \\
\hline 3 & 5.66 & 6.10 & 4.59 & 5.27 & 5.62 & 5.23 \\
\hline 4 & 5.75 & 6.23 & 4.66 & 4.48 & 7.03 & 6.55 \\
\hline 5 & 5.79 & 5.25 & 4.74 & 5.04 & 6.18 & 6.67 \\
\hline 6 & 6.07 & 6.69 & 5.13 & 5.71 & 6.40 & 5.59 \\
\hline 7 & 8.37 & 8.70 & 6.83 & 7.21 & 8.45 & 8.89 \\
\hline 8 & 7.41 & 6.79 & 6.11 & 5.51 & 7.98 & 7.14 \\
\hline 9 & 7.01 & 7.66 & 5.88 & 6.72 & 7.80 & 8.49 \\
\hline 10 & 8.88 & 8.82 & 7.16 & 7.13 & 9.45 & 9.13 \\
\hline
\end{tabular}
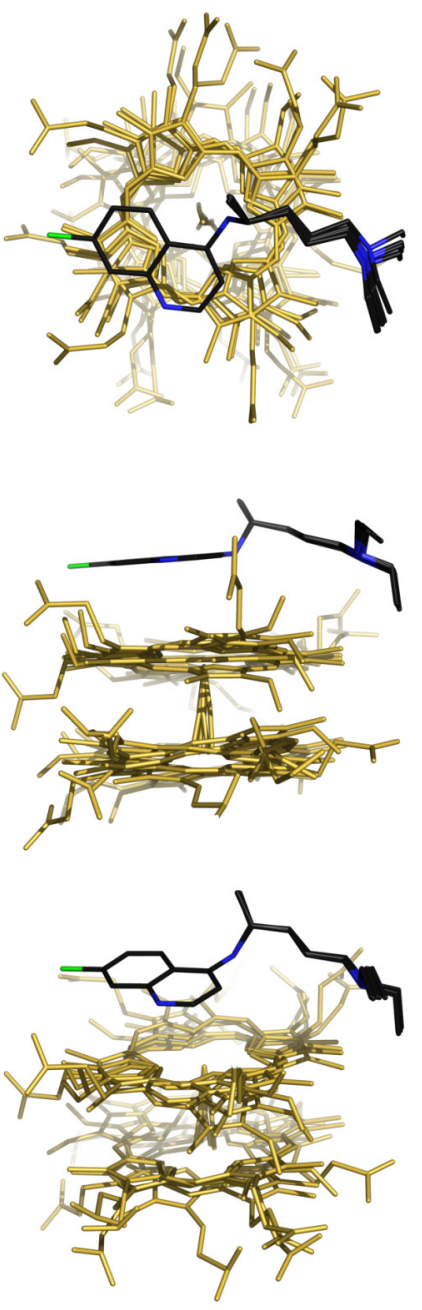

(a) FPIX:CQ pH 6.5

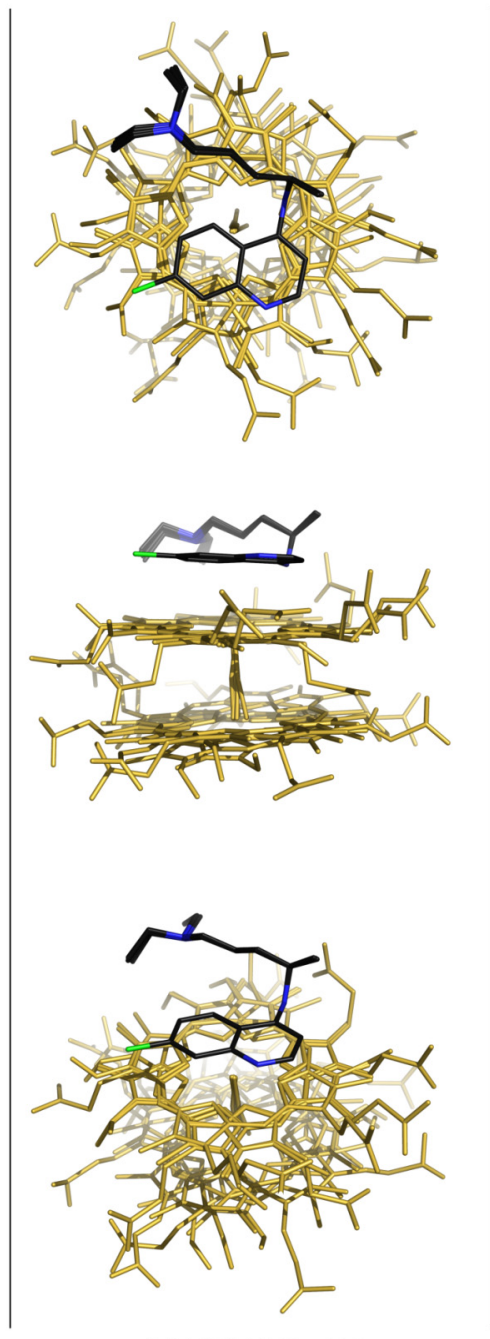

(b) FPIX:CQ pH 9

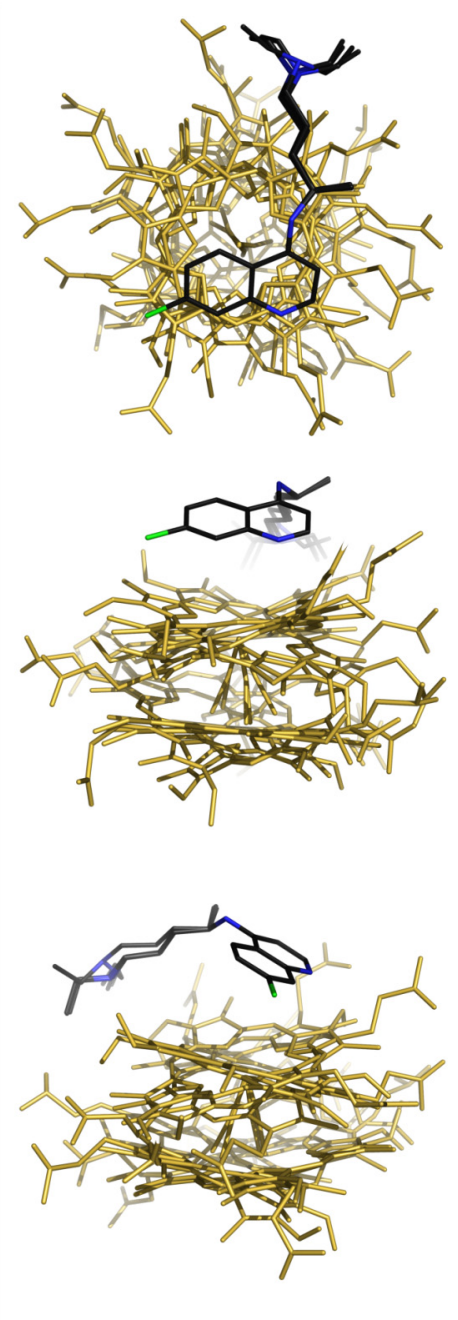

(c) FPIX:CQ in MeOD

Fig. (6). 3D models of FPIX:CQ pH 6.5 (a), pH 9 (b), and in $\mathrm{MeOD} / \mathrm{D}_{2} \mathrm{O}$ (c) in top (top row), side (middle row), and isometric view (bottom row). 
Overall stoichiometry in the complex was found to be two FPIX monomer molecules per one CQ. This confirms results from previous investigations with NMR and CD [7, 24]. Both studies, however, are also in perfect agreement with our proposed 4:2 complex, since none of the previous techniques can distinguish between a 2:1 complex and multiples thereof. Other stoichiometries as proposed in earlier studies [17], however, seem unlikely considering our Job plot and MS experiments. Additional support originates from the observation of a linear increase of relaxation rates with increasing concentrations of FPIX (Fig. S2). As already pointed out in [7], the association of one FPIX Ā-oxo dimer and one CQ molecule is a second-order reaction, resulting in a linear dependence between the FPIX concentration and the relaxation rates. This is also true for our proposed 4:2 complex if both dimer subunits are treated as independent binding sites for CQ.

None of the performed experiments, including the MS analysis, showed any indication of the existence of a FPIX:CQ 2:2 complex. The presence of CQ on one side of the FPIX $\bar{A}-$ oxo dimer seems to introduce an asymmetry in the complex, which prevents a second CQ molecule from binding on the opposite side. The FPIX:CQ 2:1 complex found in the MS analysis ([CQ-FPIX $2+\mathrm{H}]^{+}, m / z$ 1550) is most likely a fragment of the 4:2 complex. The potential existence of intermediates during complex formation requires further investigation and may provide additional information on the binding mode of CQ.

The observation of an average spin state $S=3 / 2$ for free FPIX (Fig. 1) is in contrast to a previous investigation where $S$ $=1 / 2$ was found for free FPIX at $\mathrm{pH} 6.5$ [7]. This difference may originate from slightly different sample preparation procedures. However, our results were reproduced at $\mathrm{pH} 6.5$ and $\mathrm{pH}$ 9. With $n=1.0$ and $n=1.4$, the numbers of free electrons in the complexes are close to the previous result of $n$ $=1.2$. The differences may originate from self-association of CQ [25], depending on sample preparation and $\mathrm{pH}$.

The observed change in spin state is the major evidence for formation of a 4:2 complex. While it cannot be ruled out that this observation might be explained by other hypothetical models, the ESI-MS analysis provides additional evidence for the existence of a 4:2 complex in solution. Although MS only shows that the 4:2 complex is present among other adducts, the observation of a twelvefold H/D exchange substantiates our model of mutual coordination of two Â-oxo dimers. Taken together all experimental results corroborate the assumption that the proposed 4:2 complex is formed in solution and represents the dominant species at millimolar concentrations as used for the NMR experiments.

Regarding its biological function, the 4:2 complex displays an interesting feature: Atoms $\mathrm{Fe}^{(\mathrm{b})}$ and $\mathrm{Fe}^{(\mathrm{c})}$ in Fig. (3) are mutually coordinated by carboxy groups of the neighboring $\bar{A}-$ oxo dimer. This type of coordination is identical to . -hematin (malaria pigment), the in vivo crystallized form of FPIX [6, 26]. Thus, the double dimer may function as an important precursor to hemozoin crystallization in the parasite. The antimalarial properties of CQ may arise from noncovalent binding on both sides of the FPIX double dimer, thereby blocking further crystallization of FPIX into . -hematin by the malaria parasite [27, 28]. High concentrations of the drug, which are required for such a mode of action have been reported to accumulate in the food vacuole of the parasites [28].
The dominant attractive force in the FPIX:CQ complex is $\pi-\pi$ stacking. Development of improved antimalarial pharmacophores may enhance these driving forces by direct ionic or covalent interaction between drug molecule and FPIX. Such enhancement may possibly be achieved by introduction of an electronegative domain in the drug molecule, e.g. a central nitrogen atom, capable of interacting with the Fe center in FPIX. A first step in this direction may be represented by the recently described $\mathrm{N}, \mathrm{C}$-coupled naphthylisoquinoline alkaloids [29], which show promising activity against plasmodium.

Free complexes can exercise a twofold mode of action. Free FPIX exerts strong oxidative stress on lipid membranes like those of the digestive vacuole of the parasite. Since Plasmodium does not have other pathways of FPIX detoxification [30], free FPIX can lead to membrane destruction and death of the parasite. Secondly, the presence of CQ leads to stronger association of FPIX to the membrane and thus increases its toxic effect on Plasmodium.

\section{SUPPORTING INFORMATION}

Table S1. Calculated Relaxation Rates $R$ of the FPIX:CQ Complex (pH 6.5) at 9.4 T (400 MHz), 14.1 T (600 $\mathrm{MHz})$, and 17.6 T (750 MHz) Magnetic Field Strength

\begin{tabular}{|c|c|c|c|}
\hline Atom & $\mathbf{R}[\mathbf{1} / \mathbf{s}] \mathbf{4 0 0} \mathbf{~ M H z}$ & $\mathbf{R}[\mathbf{1} / \mathbf{s}] \mathbf{6 0 0} \mathbf{~ M H z}$ & $\mathbf{R}[\mathbf{1} / \mathbf{s}] \mathbf{7 5 0} \mathbf{~ M H z}$ \\
\hline \hline 1 & 196 & 172 & 185 \\
\hline 2 & 193 & 169 & 142 \\
\hline 3 & 236 & 214 & 189 \\
\hline 4 & 213 & 198 & 169 \\
\hline 5 & 209 & 173 & 177 \\
\hline 6 & 209 & 122 & 137 \\
\hline 7 & 23.3 & 20.6 & 17.4 \\
\hline 8 & 47.0 & 41.9 & 38.1 \\
\hline 9 & 61.5 & 54.2 & 61.0 \\
\hline 10 & 15.4 & 13.8 & 13.4 \\
\hline
\end{tabular}

Table S2. Calculated Relaxation Rates $R$ of the FPIX:CQ Complex (pH 9) at 9.4 T (400 MHz), 14.1 T (600 MHz), and 18.8 T $(800 \quad$ MHz $)$ Magnetic Field Strength. Peaks 1 and 2 were Not Resolved in the Spectrum and Were Discarded in the Following Calculations

\begin{tabular}{|c|c|c|c|}
\hline Atom & $\mathbf{R}[\mathbf{1} / \mathbf{s}] \mathbf{4 0 0} \mathbf{~ M H z}$ & $\mathbf{R}[\mathbf{1} / \mathbf{s}] \mathbf{6 0 0} \mathbf{~ M H z}$ & $\mathbf{R}[\mathbf{1} / \mathbf{s}] \mathbf{7 5 0} \mathbf{~ M H z}$ \\
\hline \hline $1+2$ & 633 & 567 & 479 \\
\hline 3 & 848 & 846 & 569 \\
\hline 4 & 773 & 692 & 565 \\
\hline 5 & 713 & 568 & 553 \\
\hline 6 & 465 & 225 & 534 \\
\hline 7 & 77.9 & 68.1 & 59.6 \\
\hline 8 & 146 & 127 & 125 \\
\hline 9 & 158 & 139 & 209 \\
\hline 10 & 60.4 & 51.9 & 42.8 \\
\hline
\end{tabular}


Table S3. Calculated Relaxation Rates $R$ of the FPIX:CQ Complex (in MeOD/D2O (vol/vol 3:1)) at $9.4 \mathrm{~T}(400$ MHz), 14.1 T (600 MHz), and $18.8 \mathrm{~T}(800 \mathrm{MHz})$ Magnetic Field Strength

\begin{tabular}{|c|c|c|c|}
\hline Atom & $\mathbf{R}[\mathbf{1} / \mathbf{s}] \mathbf{4 0 0} \mathbf{~ M H z}$ & $\mathbf{R}[\mathbf{1} / \mathbf{s}] \mathbf{6 0 0} \mathbf{~ M H z}$ & $\mathbf{R}[\mathbf{1} / \mathbf{s}] \mathbf{8 0 0} \mathbf{~ M H z}$ \\
\hline \hline 1 & 74.5 & 77.7 & 72.1 \\
\hline 2 & 130 & 144 & 131 \\
\hline 3 & 170 & 193 & 177 \\
\hline 4 & 45.1 & 48.8 & 46.1 \\
\hline 5 & 95.8 & 110 & 97.9 \\
\hline 6 & 31.6 & 193 & 104 \\
\hline 7 & 19.9 & 14.1 & 13.3 \\
\hline 8 & 22.7 & 21.5 & 21.5 \\
\hline 9 & 23.8 & 24.1 & 27.4 \\
\hline 10 & 8.51 & 7.40 & 7.91 \\
\hline
\end{tabular}

Figure S1.

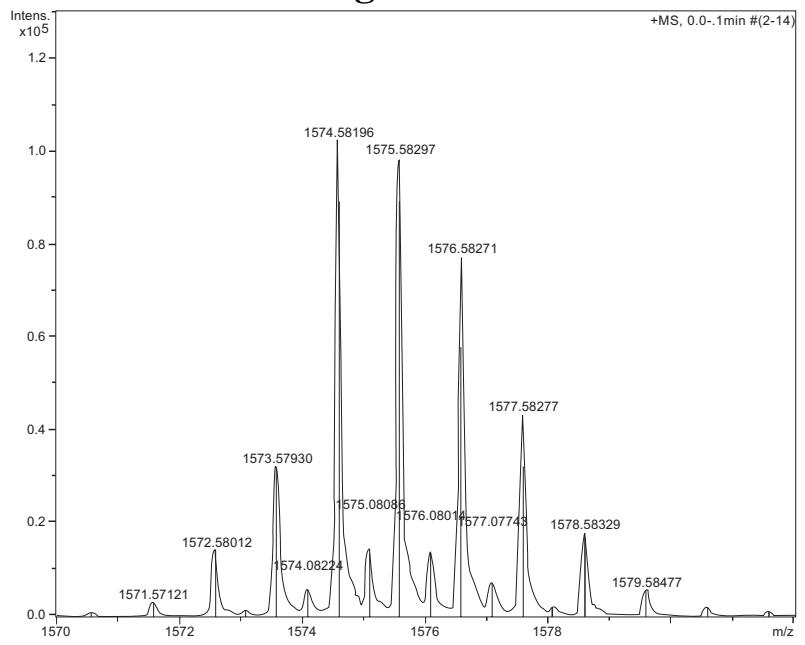

ESI-TOF mass spectrum of a $250 \overline{\mathrm{A} M} \mathrm{CD}_{3} \mathrm{OD} / \mathrm{D}_{2} \mathrm{O}$ (vol/vol $3: 1$ ) solution of the CQ:FPIX mixture. The isotope distribution at $\mathrm{m} / \mathrm{z}$ 1575.08086 reveals the formation of the double deuterated and H/D exchanged complex CQFPIX-O-FPIX ${ }_{2}-\mathrm{O}-\mathrm{FPIX}-\mathrm{CQ}$ besides the mono deuterated [CQ-FPIX-O-FPIX-CQ $+\mathrm{H}^{+}{ }^{+}$.

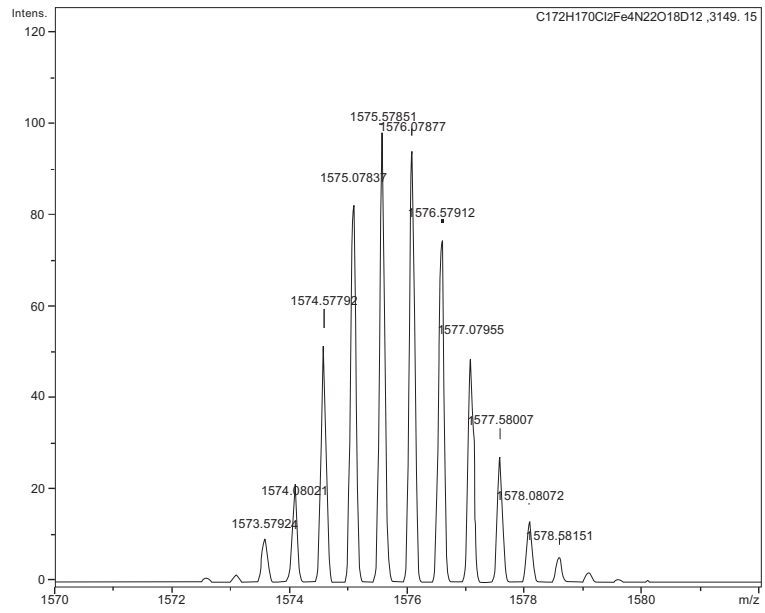

Calculated isotopic distribution of the deuterated [CQ-FPIX-OFPIX $_{2}$-O-FPIX-CQ+2D] ${ }^{2+}$, corresponding to the elemental composition $\left[\mathrm{C}_{172} \mathrm{H}_{170} \mathrm{Cl}_{2} \mathrm{Fe}_{4} \mathrm{~N}_{22} \mathrm{O}_{18} \mathrm{D}_{12}\right]$

\section{Figure S2.}

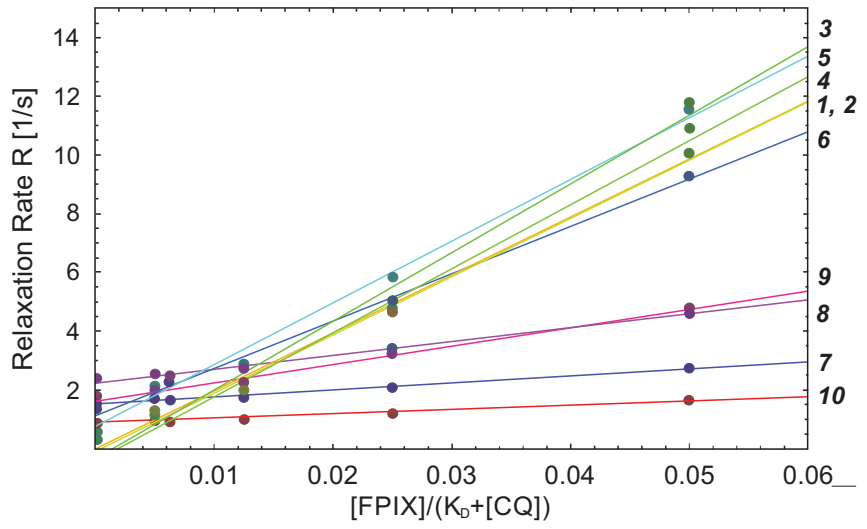

Determination of the relaxation rates: Example plot ( $\mathrm{pH} 6.5,750$ $\mathrm{MHz}$ ) of the longitudinal relaxation rate $\mathrm{R}$ of all resonances (indicated on the right margin) in the spectrum versus the concentration of FPIX in the sample: $[\mathrm{FPIX}] /\left(\mathrm{K}_{\mathrm{D}}+[\mathrm{CQ}]\right)$. [CQ] was held constant at $2 \mathrm{mM}$ and [FPIX] was increased during the NMR titration. The paramagnetic effect causes $\mathrm{R}$ to increase with [FPIX]. No error bars are indicated, since each relaxation rate was determined with high precision. The main error source in the extrapolated relaxation rates is due to uncertainties of the concentration.

\section{Figure S3.}

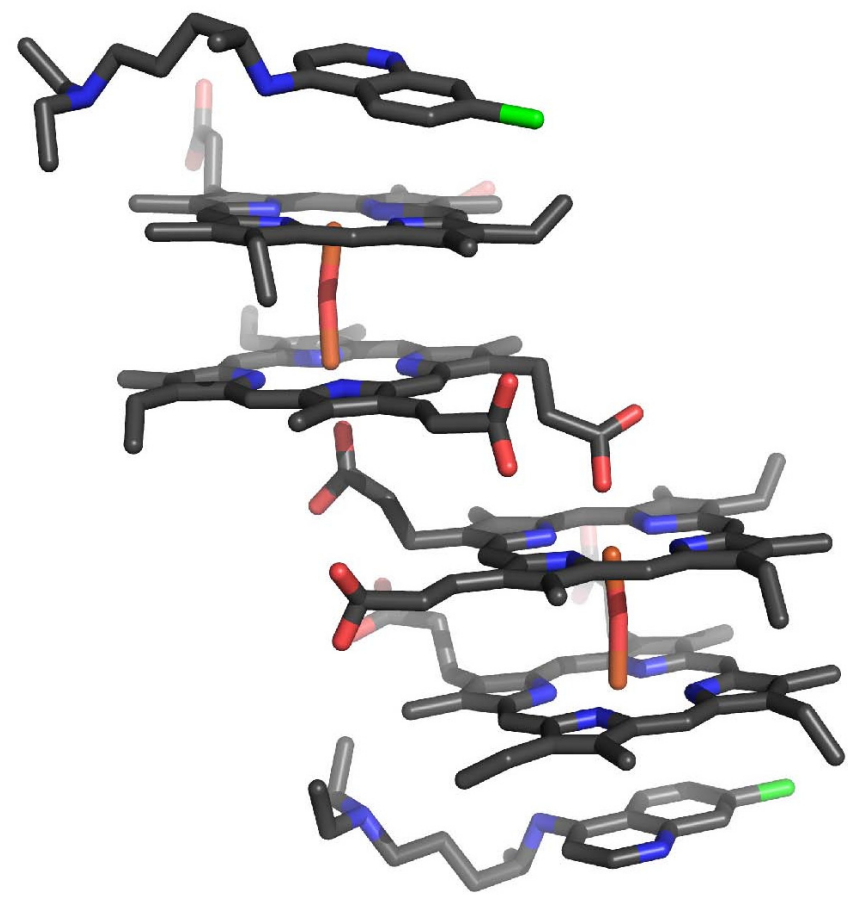

Illustration of the proposed FPIX:CQ 4:2 complex: Two FPIX Aoxo dimers are mutually coordinated by one carboxy group of the corresponding subunit of the second $\bar{A}$-oxo dimer. The four FPIX monomer subunits bind two CQ molecules.

\section{ACKNOWLEDGMENTS}

This work was supported by the Deutsche Forschungsgemeinschaft (SFB630 "Novel Agents against Infectious Diseases", projects A2 and C2). We acknowledge the Fonds der Chemischen Industrie for granting a fellowship (K.F.S.) 
and supplies (G.B.). We thank Dr. Matthias Grüne, Prof. Paul Rösch, and Dr. Kristian Schweimer for making spectrometer time available.

\section{REFERENCES}

[1] Snow RW, Guerra CA, Noor AM, Myint HY, Hay SI. The global distribution of clinical episodes of Plasmodium falciparum malaria. Nature 2005; 434: 214-217.

[2] Loeb RF, Clarke WM, Coateney GR, et al. Activity of a New Antimalarial Agent, Chloroquine (SN 7618). J Am Med Assoc 1946; 130: 1069-1070.

[3] Foley M, Tilley L. Quinoline antimalarials: Mechanisms of action and resistance. Int J Parasitol 1997; 27: 231-240.

[4] Cheng L, Lee J, Powell DR, Richter-Addo GB. mu-oxobis[(protoporphyrin IX dimethyl ester)iron(III)]. Acta Crystallogr Sect E: Struct Rep Online 2004; 60: M1340-M1342.

[5] Silver J, Lukas B. Mossbauer Studies on Protoporphyrin-IX Iron(III) Solutions. Inorg Chim Acta 1983; 78: 219-224.

[6] Pagola S, Stephens PW, Bohle DS, Kosar AD, Madsen SK. The structure of malaria pigment beta-haematin. Nature 2000; 404: $307-$ 310.

[7] Leed A, DuBay K, Ursos LMB, Sears D, de Dios AC, Roepe PD. Solution structures of antimalarial drug-heme complexes. Biochemistry 2002; 41: 10245-10255.

[8] Schwedhelm KF, Horstmann M, Faber JH, Reichert Y, Bringmann $\mathrm{G}$, Faber C. The novel antimalarial compound dioncophylline $\mathrm{C}$ forms a complex with heme in solution. ChemMedChem 2007; 2: 541-548.

[9] Solomon I. Relaxation Processes in a system of 2 spins. Phys Rev 1955; 99: 559-565.

[10] Maccarthy P. Simplified experimental route for obtaining job's curves. Anal Chem 1978; 50: 2165-2165.

[11] Hirose K. A practical guide for the determination of binding constants. J Inclusion Phenom Macrocycl Chem 2001; 39: 193-209.

[12] Evans DF. The determination of the paramagnetic susceptibility of substances in solution by nuclear magnetic resonance. J Am Chem Soc $1959 ; 2003-2005$

[13] Crawford TH, Swanson J. Temperature dependent magnetic measurements and structural equilibria in solution. J Chem Educ 1971; 48: 382-386.

[14] Deutsch JL, Poling SM. Determination of paramagnetic susceptibility by NMR - a physical chemistry experiment. J Chem Educ 1969; 46: 167-168.

[15] Theisen RM, Shearer J, Kaminsky W, Kovacs JA. Steric and electronic control over the reactivity of a thiolate-ligated $\mathrm{Fe}(\mathrm{II})$ complex with dioxygen and superoxide: Reversible mu-oxo dimer formation. Inorg Chem 2004; 43: 7682-7690.

[16] Bertini I, Luchinat C, Parigi G. Solution NMR of Paramagnetic Molecules. Elsevier: New York, 2001.

[17] Moreau S, Perly B, Biguet J. Interactions between Chloroquine and Ferriprotoporphyrine IX. Nuclear Magnetic-Resonance Study. Biochimie 1982; 64: 1015-1025.

[18] Schwieters CD, Kuszewski JJ, Tjandra N, Clore GM. The XplorNIH NMR molecular structure determination package. J Magn Reson 2003; 160: 65-73.

[19] DeLano WL. The PyMOL Molecular Graphics System. DeLano Scientific: San Carlos, CA, 2002.

[20] Dascombe MJ, Drew MGB, Morris H, et al. Mapping antimalarial pharmacophores as a useful tool for the rapid discovery of drugs effective in vivo: Design, construction, characterization, and pharmacology of metaquine. J Med Chem 2005; 48: 5423-5436.

[21] Egan TJ, Hunter R, Kaschula CH, Marques HM, Misplon A, Walden J. Structure-function relationships in aminoquinolines: Effect of amino and chloro groups on quinoline-hematin complex formation, inhibition of beta-hematin formation, and antiplasmodial activity. J Med Chem 2000; 43: 283-291.

[22] Vippagunta SR, Dorn A, Bubendorf A, Ridley RG, Vennerstrom JL. Deferoxamine: Stimulation of hematin polymerization and antagonism of its inhibition by chloroquine. Biochem Pharmacol 1999; 58: 817-824.

[23] Mitchell PR, Parish RV. 18-electron rule. J Chem Educ 1969; 46: 811-814

[24] Blauer G, Akkawi M, Fleischhacker W, Hiessböck R. Chirality 1997; 10: 556.

[25] Casabianca LB, de Dios AC. C-13 NMR study of the selfassociation of chloroquine, amodiaquine, and quinine. J Phys Chem A 2004; 108: 8505-8513.

[26] Buller R, Peterson ML, Almarsson O, Leiserowitz L. Quinoline binding site on malaria pigment crystal: a rational pathway for antimalaria drug design. Cryst Growth Des 2002; 2: 553-562.

[27] Egan TJ, Ncokazi KK. Quinoline antimalarials decrease the rate of beta-hematin formation. J Inorg Biochem 2005; 99: 1532-1539.

[28] Foley M, Tilley L. Quinoline antimalarials: Mechanisms of action and resistance and prospects for new agents. Pharmacol Ther 1998; 79: 55-87.

[29] Bringmann G, Kajahn I, Reichert M, et al. Ancistrocladinium A and $\mathrm{B}$, the first $\mathrm{N}, \mathrm{C}$-coupled naphthyldihydroisoquinoline alkaloids, from a congolese ancistrocladus species. J Org Chem 2006; 71: 9348-9356.

[30] Loria P, Miller S, Foley M, Tilley L. Inhibition of the peroxidative degradation of haem as the basis of action of chloroquine and other quinoline antimalarials. Biochem J 1999; 339: 363-370.

(c) Schwedhelm et al.; Licensee Bentham Open.

This is an open access article distributed under the terms of the Creative Commons Attribution License (http://creativecommons.org/license/by/2.5/), which permits unrestrictive use, distribution, and reproduction in any medium, provided the original work is properly cited. 